\title{
Gamma-Ray Bursts in the Era of Rapid Followup
}

\author{
C. G. Mundell, ${ }^{1}$ C. Guidorzi, ${ }^{1,2}$ and I. A. Steele ${ }^{1}$ \\ ${ }^{1}$ Astrophysics Research Institute, Liverpool John Moores University, Twelve Quays House, Birkenhead CH41 1LD, UK \\ ${ }^{2}$ Physics Department, University of Ferrara, via Saragat 1, 44122 Ferrara, Italy
}

Correspondence should be addressed to C. Guidorzi, guidorzi@fe.infn.it

Received 30 June 2009; Revised 9 February 2010; Accepted 5 March 2010

Academic Editor: Joshua S. Bloom

Copyright ( 2010 C. G. Mundell et al. This is an open access article distributed under the Creative Commons Attribution License, which permits unrestricted use, distribution, and reproduction in any medium, provided the original work is properly cited.

\begin{abstract}
We present a status report on the study of gamma-ray bursts (GRBs) in the era of rapid followup using the world's largest robotic optical telescopes-the $2 \mathrm{~m}$ Liverpool and Faulkes telescopes. Within the context of key unsolved issues in GRB physics, we describe (1) our innovative software that allows real-time automatic analysis and interpretation of GRB light curves, (2) the novel instrumentation that allows unique types of observations (in particular, early time polarisation measurements), and (3) the key science questions and discoveries to which robotic observations are ideally suited, concluding with a summary of current understanding of GRB physics provided by combining rapid optical observations with simultaneous observations at other wavelengths.
\end{abstract}

\section{Introduction}

Gamma-Ray Bursts (GRBs) are the most powerful explosions in the Universe and, arguably, represent the most significant new astrophysical phenomenon since the discovery of quasars and pulsars. As their name suggests, GRBs are detected as brief, intense and totally unpredictable flashes of high-energy gamma rays, thought to be produced during the core collapse of massive stars (long-soft bursts, $T_{\gamma}>2$ seconds) or the merger of two compact objects such as two neutron stars or a neutron star and a stellarmass black hole (short-hard bursts, $T_{\gamma}<2$ seconds). Although discovered through their $\gamma$-ray emission [1], they are now known to emit nonthermal radiation detectable across the electromagnetic spectrum [2-4]. However, despite their enormous luminosity, their unpredictability and short duration limit rapid, accurate localisation and observability with traditional telescopes. Consequently, new ground and space-based facilities have been developed over the past decade; dedicated satellites optimised for GRB detection and followup, such as Swift [5], are revolutionizing GRB studies by locating $\sim 100$ bursts per year with $\gamma$-ray positions accurate to $\sim 3^{\prime}$ and X-ray positions accurate to $5^{\prime \prime}$ within seconds or minutes of the burst. Here we describe the automatic ground-based followup of GRBs with the world's largest robotic optical telescopes that use intelligent software and innovative instruments.

The Era of Rapid Followup: Predictions and Outcomes. Before the launch of current satellites such as Swift, Integral, and Fermi, significant progress in understanding GRBs had been made since their discovery, in particular the general $\gamma$ and $\mathrm{X}$-ray properties. The first crucial step in disseminating realtime GRB positions to ground observers was triggered by BATSE on the CGRO [6] through the GRB Coordinates Network (GCN) [7] via internet socket connection (no humans-in-the-loop). This drove development of the first generation of wide-field robotic followup ground-based facilities, such as GROCSE, ROTSE, and LOTIS, culminating with the discovery of the optical flash associated with GRB 990123 [8]. BATSE provided an invaluable catalogue of prompt $\gamma$-ray profiles, whose isotropic sky distribution and inhomogeneous intensity distribution suggested a cosmological origin [6], and BeppoSAX [9] revolutionised the cosmological study of GRBs by providing subarcmin $\left(\sim 50^{\prime \prime}\right)$ localisation of X-ray afterglows that enabled latetime ( hours) optical followup with traditional groundbased telescopes and redshift determinations. Collimation of the ejecta (i.e., jets) was inferred from temporal breakssteepening — of optical light curves at $\sim 1$ day post burst and 


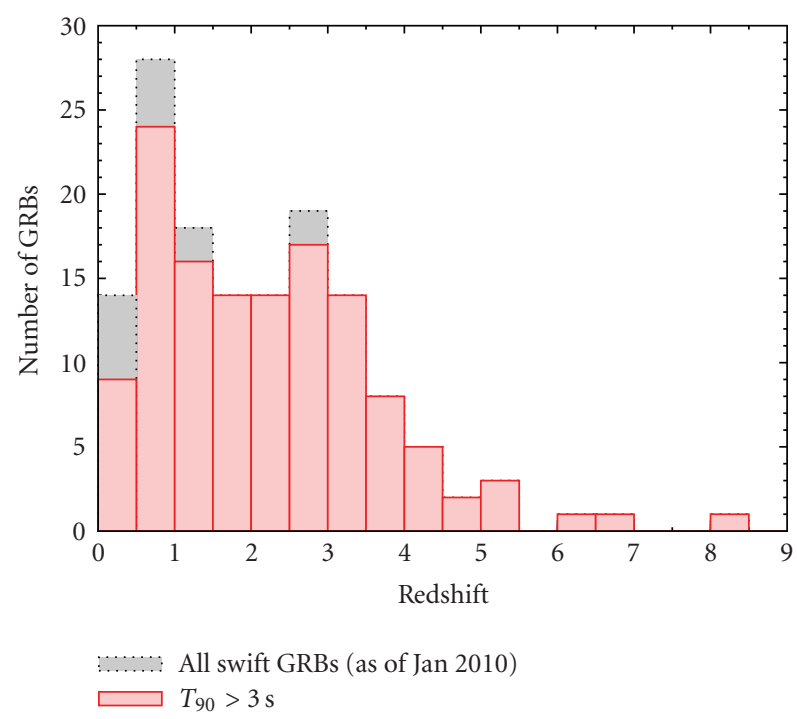

FIgURE 1: Redshift distribution of Swift GRBs detected to-date.

the concept of a universal central engine and the use of GRBs as standardisable cosmological candles was introduced $[10,11]$.

The possibility for great advances with the launch of Swift was fully recognised. Optical counterparts were expected to be found for all GRBs with many GRBs expected to exhibit bright optical flashes from reverse shock emission at early-times, similar to GRB 990123 [8]. An increase in the number of GRBs detected would lead to many jet breaks being identified, short GRBs would be easily observed and understood, and identification of GRBs at very high redshift would be routine. Instead, 50\% of GRBs remain optically dark, despite deep, rapid followup [12-15]; there is a dearth of bright reverse-shock optical emission [16]; light curves are complex in all bands with a variety of chromatic and achromatic breaks and flares observed (e.g., [17-23]). Jet breaks have proven elusive, short bursts remain technically challenging [24], and only 3 GRBs have been identified to lie a $z>6$ (Figure 1) [25-28].

\section{Robotic Followup and Intelligent Autonomy}

The field of GRB research is the most rapidly evolving topic in modern day astrophysics_-driven primarily by technological innovation in both hardware and software; most notable is the need for rapid, intelligent, and fully autonomous followup. Within the context of robotic telescopes devoted to searching for optical counterparts to GRBs (e.g., [29-36]), the Liverpool Telescope (LT) offers a unique combination of sensitivity, speed, instrument choice, and real-time reduction pipeline with complexity and flexibility. The LT, owned and operated by Liverpool John Moores University (LJMU), has a $2 \mathrm{~m}$ diameter mirror, altitude-azimuth design, final focal ratio f/10, a comprehensive suite of instruments, and a fully robotic control system. As shown in Figure 2, it is sited at the Observatorio del Roque de los Muchachos in La Palma. Optimised for robotic followup of transient sources, the LT
TABLE 1: Current instrumentation on the Liverpool and Faulkes telescopes and associated GRB science goals; upcoming instrumentation is shown in italics.

\begin{tabular}{|c|c|}
\hline Instrumentation & Science goals \\
\hline \multirow{4}{*}{$\begin{array}{l}\text { Optical Camera }\left(\mathrm{FoV} \sim 5^{\prime}\right) \\
\left(\mathrm{BVRi} z^{\prime}\right)(\mathrm{LT} / \mathrm{FTN} / \mathrm{FTS})\end{array}$} & (i) Early multicolour light curves \\
\hline & (ii) Shock physics/ISM studies \\
\hline & $\begin{array}{l}\text { (iii) Later-time light curves/jet } \\
\text { breaks }\end{array}$ \\
\hline & (iv) GRB-supernova connection \\
\hline \multirow[t]{2}{*}{$\begin{array}{l}\text { RINGO2 polarimeter }(\mathrm{FoV} \\
\left.\sim 4^{\prime}\right) \text { (LT only) }\end{array}$} & $\begin{array}{l}\text { (i) Early-time polarisation } \\
\text { studies } 1 \% \text { polarisation at } \\
r^{\prime}<17 \text { mag in } 1 \text { minute }\end{array}$ \\
\hline & $\begin{array}{l}\text { (ii) Fundamental tests of } \\
\text { magnetization }\end{array}$ \\
\hline \multirow{2}{*}{$\begin{array}{l}\text { SupIRCam Infrared Camera } \\
\left(\text { FoV } \sim 1^{\prime}\right) \text { (LT only) }\end{array}$} & (i) High- $z$ "naked" bursts \\
\hline & (ii) Low- $z$ "obscured" bursts \\
\hline $\begin{array}{l}\text { FRODOSpec IFU (FoV } \\
\left.\sim 11^{\prime \prime}\right) \text { (LT only) } \\
\end{array}$ & $\begin{array}{l}\text { (i) Early evolution of } \\
\text { circumburst medium }\end{array}$ \\
\hline $\begin{array}{l}\left.\text { STILT (FoV } 1^{\circ} / 20^{\circ} / 180^{\circ}\right)(\mathrm{LT} \\
\text { only) }\end{array}$ & $\begin{array}{l}\text { (i) Bright bursts/neutrino } \\
\text { counterparts }\end{array}$ \\
\hline $\begin{array}{l}\text { IO_wide-field optical/IR } \\
\text { imager (LT-2010) }\end{array}$ & $\begin{array}{l}\text { (i) Deep, simultaneous optical/IR } \\
\text { light curves over } 2^{\circ} \mathrm{FoV}\end{array}$ \\
\hline
\end{tabular}

was designed to have a fully open enclosure, robustness to wind gusts, and a fast slew rate of $2 \%$; currently as the fastest telescope for its size, it observes GRBs within 1-3 minutes of receipt of a satellite alert. Although smaller robotic telescopes slew more quickly, the LT is more sensitive to fainter bursts at early-time. Most importantly, the LT can perform unique early-time polarisation measurements (see Section 4).

The LT has five instrument ports: four folded and one straight-through, selected automatically within 30 seconds by a deployable rotating mirror in the Acquisition and Guidance (AG) box. Table 1 describes the instruments available and the related GRB science goals. All GRB followups with the LT begin with RINGO polarimetry exposures before continuing with a sequence of 10-s $R$-band exposures that are used to automatically identify an optical counterpart, determine its characteristics, and conduct subsequent optimized followup observations with the most appropriate instrument. Guidorzi et al. [36] describe the intelligent software logic-LT-TRAP_-developed to perform the real time analysis and followup. Automatic multicolour light curves of optical transients brighter than $R \sim 19$ mag are produced in real-time; transients brighter than $R \sim$ 15 mag trigger additional RINGO polarisation observations before continuing with multicolour imaging. Faint OTs or nondetections trigger deep exposures in red filters. The $2 \mathrm{~m}$ Faulkes telescopes ${ }^{1}$, clones of the LT, provide additional sky coverage, operate the same intelligent LT-TRAP softwareapart from small differences due to the different instruments mounted on each facility - and concentrate on multicolour optical imaging (Figure 2 and Table 1). Figure 3 displays the flow chart of the LT-TRAP. 


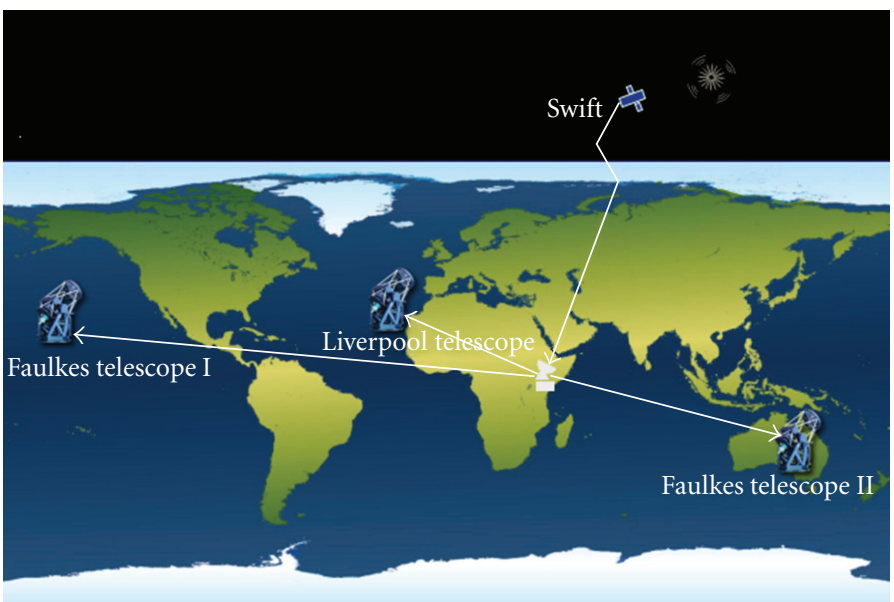

FIgURE 2: The locations of the $2 \mathrm{~m}$ robotic Liverpool Telescope and its clones the Faulkes telescopes. GRB triggers from satellites drive prompt automatic followup.

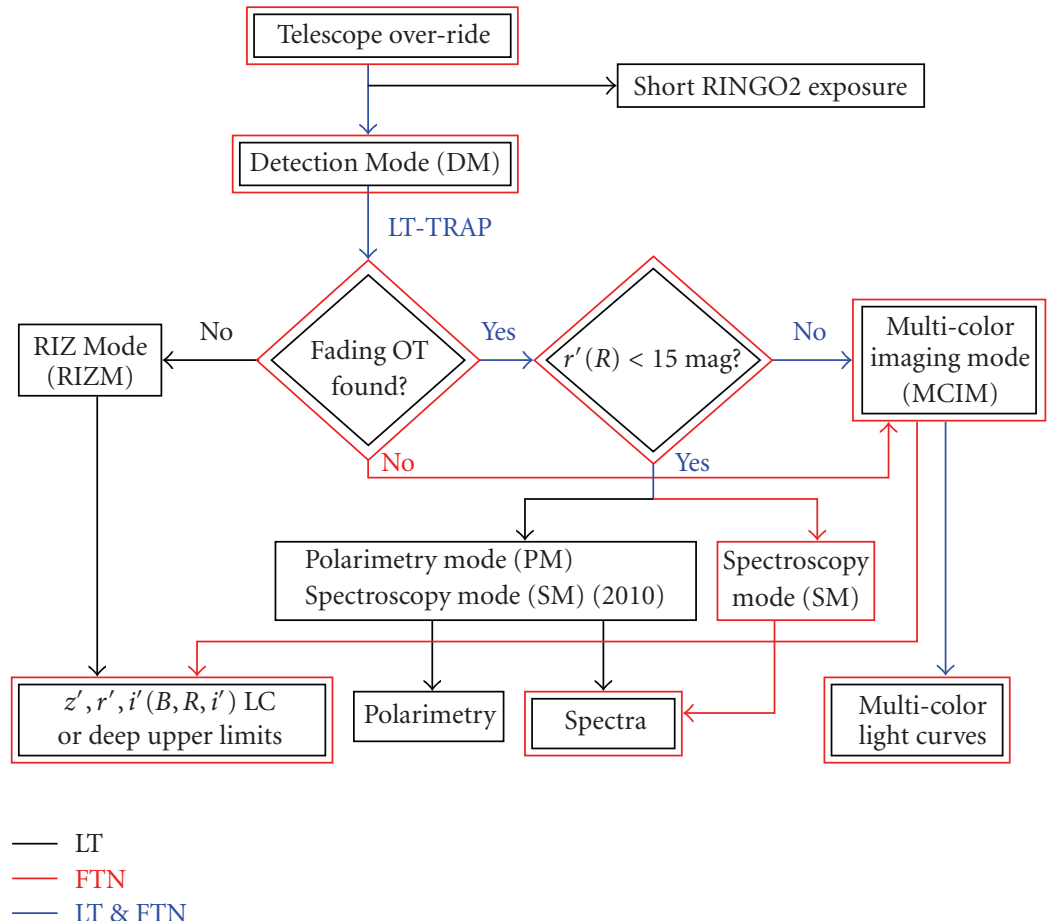

FIGURE 3: Flow chart of the robotic GRB pipeline currently running on the Liverpool and Faulkes Telescopes (adapted from [36]).

\section{Characteristics of Multiwavelength Light Curves}

A major breakthrough provided by Swift was the discovery of complex light curves at X-ray energies that led to introduction of the so-called canonical light curve [37, 38], characterized by four decay segments: (1) an "early steep decay" with power-law decay indices $\sim 3$ or even steeper; (2) a "shallow or flat decay" with a typical index around 0.5 ; (3) a normal decay, with indices around 1.2; and (4) finally, the late steep decay with typical values around 2 . In $\sim 50 \%$ of cases, flares are also superimposed
$[20,21]$. Some complexity is attributed to on-going central engine activity producing different emission components that originate from spatially distinct regions but that are temporally coincident, but alternative explanations invoking an external origin have also been suggested [39, 40]. Unlike other high-energy phenomena such as Active Galactic Nuclei, GRBs will remain spatially unresolved with current and future instrumentation. Model-dependent temporal properties therefore provide a powerful, indirect probe of the expanding fireball and its interaction with the surrounding medium. Polarisation measurements provide a direct probe. 


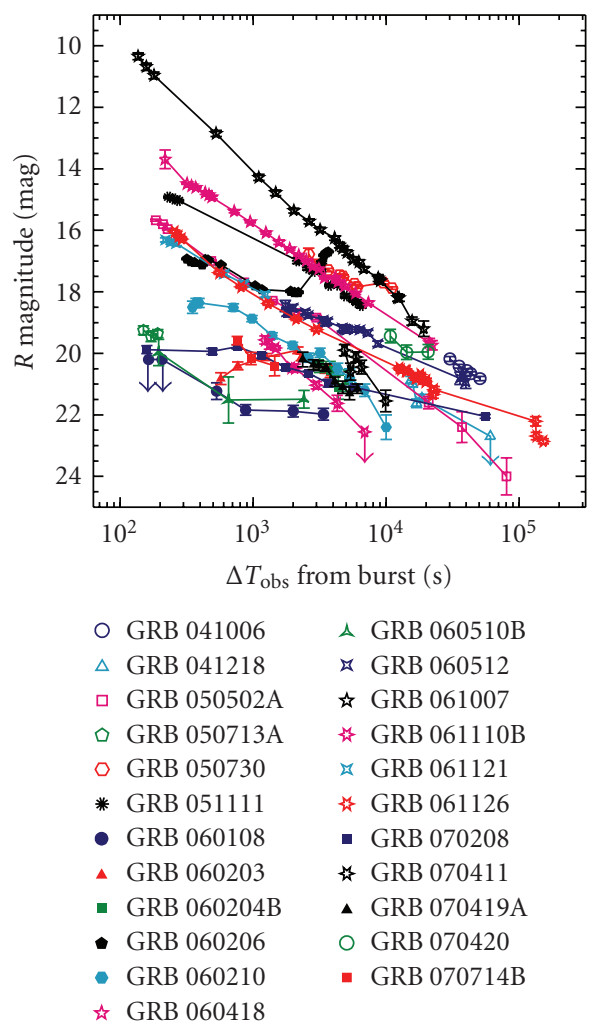

(a)
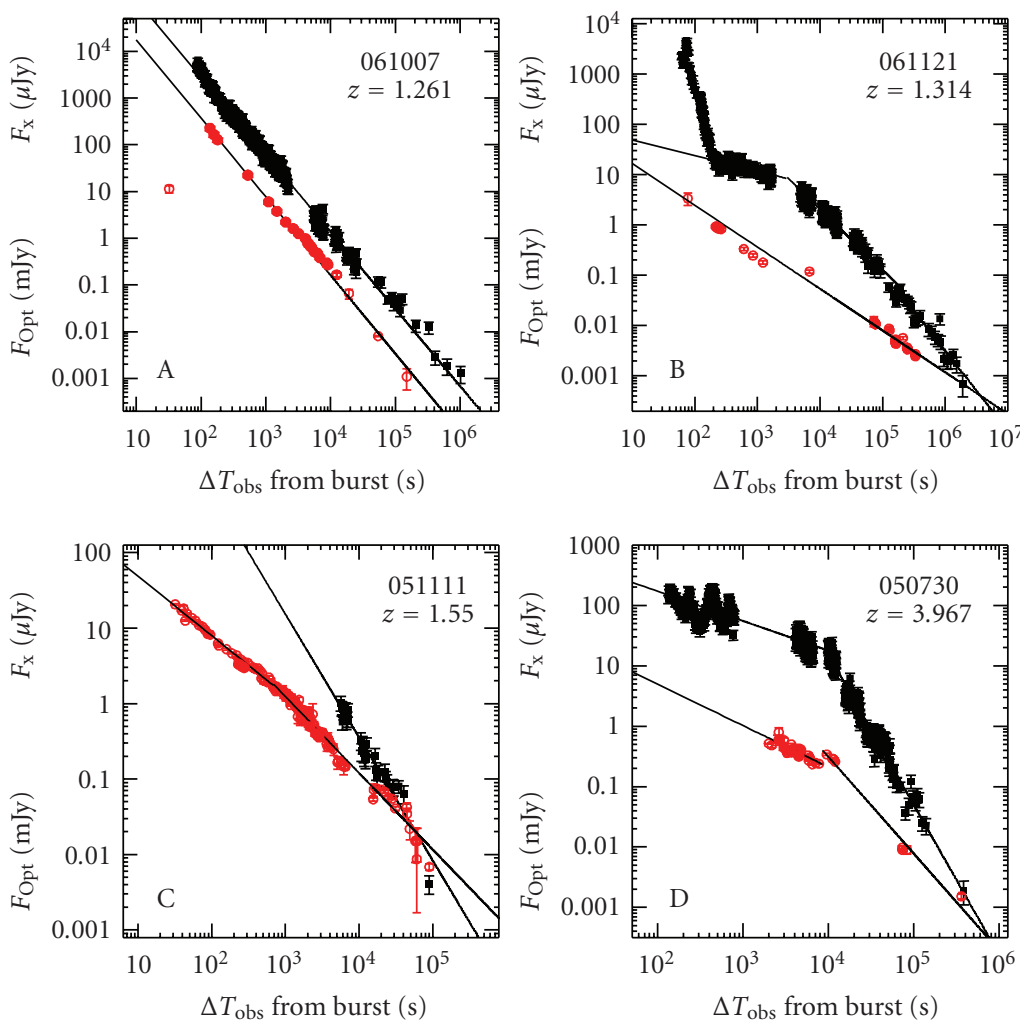

(b)

Figure 4: (a) Selection of early-time optical light curves from the Liverpool and Faulkes telescopes. (b) Examples of X-ray (black) and optical (red) light curve comparisons leading to classification scheme using synchrotron model breaks [12].

At optical wavelengths, a variety of light curve properties are expected depending on the relative contributions of emission from reverse and forward shocks, the presence of additional energy injection by a long-lived central engine, and the time of the observations with respect to the initial burst.

Melandri et al. [12] classified a sample of 63 bursts via their optical and X-ray light curves. 50\% of the sample remained optically "dark" despite rapid, deep observations through red filters. Optical counterparts ranged in brightness from $R \sim 10$ mag to $\sim 22 \mathrm{mag}$ in the first minutes after the burst and showed a range of decay behaviours (Figure 4). Flares, although less common in the optical than X-ray band, were present and in some cases (e.g., GRB 060206) showed direct evidence of significant energy injection [41-44].

By comparing optical and X-ray light curves observed from $t=100$ seconds to $\sim 10^{6}$ seconds post burst, Melandri et al. [12] introduced a coherent classification of optical/Xray light curves under the framework of the standard fireball model and synchrotron theory, using the presence or absence of temporal breaks in each band (Figure 4); the temporal location and evolution of chromatic and achromatic breaks depend on typical synchrotron and cooling frequencies with respect to the observing bands. Simply, class A shows no break in optical or X-ray bands because $v_{c}$ lies above or between the two bands; class B shows a break in the X-ray but not in the optical band as $v_{c}$ passes through the X-ray band; class $\mathrm{C}$ shows a break in the optical but not the Xray band due to $v_{c}$ passing through the optical band; class $D$ has breaks in both bands as energy injection stops or a jet break occurs. $60 \%$ of optically detected bursts were explained with the forward shock model, while the remainder required energy injection and/or an ambient density gradient. GRB 070419A required a long-lived central engine ( $\sim 250$ seconds in $\gamma$ and X-rays), a finely tuned energy injection rate and an abrupt cessation of injection (Figure 5).

3.1. Long-Lived Central Engines Explain Bright and Dark Bursts. Large robotic telescopes such as the LT provide deep upper limits at early-times (e.g., $R<22 \mathrm{mag}$ ), thereby ruling out slow followup as a reason for nondetection of optical afterglows. Observing with red filters, the LT and FTs can identify GRBs at $z<4$ within the first few minutes after the burst via $R$-band photometric dropouts; a deep, early upper limit in $R$-band plus an $i^{\prime}$-band detection quickly identified GRB 060927 as a moderately high-redshift source $(z=5.467)$ $[46,47]$. Whilst a small fraction of optically undetected GRBs may lie at very high redshift so have their rest frame emission redshifted out of optical observing bands (e.g., GRB 090423 at $z=8.1[27,28]$ ), others require a physical explanation for their darkness. Some may be explained by a relatively flat optical-to-X-ray spectral index and modest dust extinction (e.g., GRB 060108 [48]; for typical extinction values, see, e.g., $[49,50])$. The underlying physical mechanism may be energy 


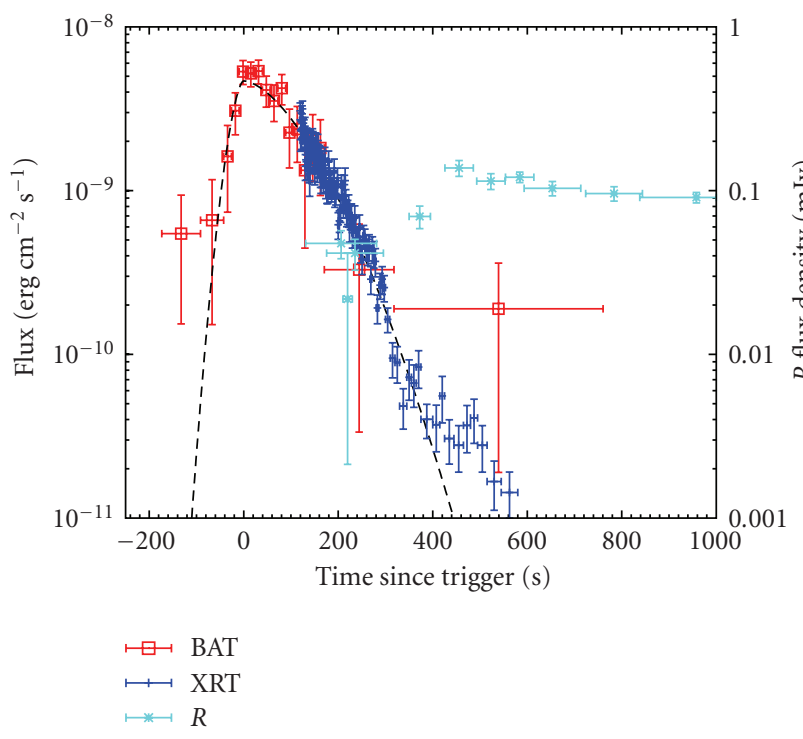

(a)

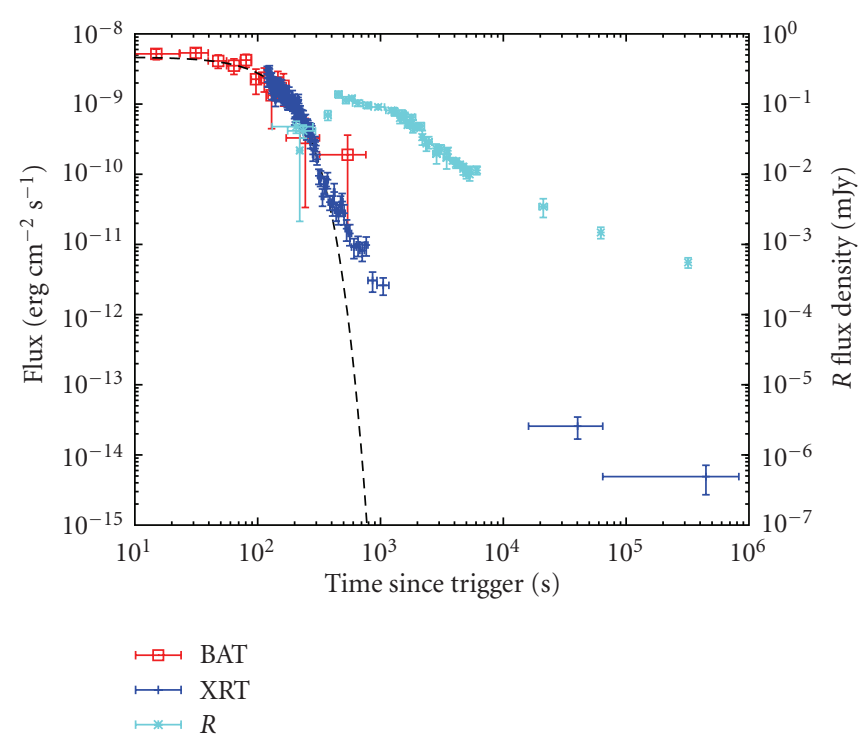

(b)

Figure 5: $\gamma$-ray, X-ray, and optical light curves of GRB 070419A. (a) Early-time light curves plotted on linear-log scale. (b) Later time evolution included and shown on log-log scale (see Melandri et al. [45]).

injection; as discussed above, a long-lived central engine is required to explain the properties of bright bursts but such a model may also provide a self-consistent explanation for dark bursts at intermediate redshift $(z<6)$. Enhanced X-ray emission in the early-time X-ray afterglow due to late-time central engine activity would make the bursts brighter than expected in the X-ray band compared to the optical band [12].

\section{Magnetized Fireballs?}

The production of synchrotron radiation requires the presence of a magnetic field but the degree of magnetization and the configuration of the magnetic fields remain a matter of debate (e.g, [51-53]). Determination of the magnetic field properties is key to understanding the driving mechanism of the explosion, namely, whether the ultrarelativistic ejecta are dominated by kinetic (baryonic) or magnetic (Poynting flux) energy. The ratio of magnetic and kinetic energy flux, $\sigma$, is used to express the magnetization (e.g., [54]) and is large if the magnetic field originates at the central engine and is advected outwards with the relativistic flow (Poynting flux jet). For a baryonic jet, $\sigma \ll 1$ and magnetic fields are assumed to be produced locally in the shock. Magnetization may be inferred from optical light curves, given a variety of assumptions, or determined directly from measurements of the degree of polarisation of the optical afterglow. In both cases, observations at the earliest possible time after the burst - when the magnetic properties of the expanding fireball are still encoded in the emission-are essential to address these issues.

Indirect Observational Tests-Light Curve Evolution. In the standard fireball model (see [55-59] for reviews) a shell of the relativistically expanding fireball collides with the circumburst medium to produce an external shock that results in the long-lived afterglow emission detectable from X-ray to optical, IR, or sometimes radio frequencies. A reverse shock propagating backwards through the shell may, in some circumstances, produce short-lived but bright optical emission-the so-called optical flash. Figure 6 shows three possible optical light curves with the relative contributions from the reverse and forward shocks identified. The strength of the reverse shock depends on magnetization and whether the typical frequency is close to the optical band $[52,54]$.

The dearth of reverse-shock optical flashes from Swift GRBs may be explained in the standard model via weak, nonrelativistic reverse shocks and a typical frequency far below the optical band at early-time; the bright optical counterpart to GRB 061007 (Figure 7) illustrates this with its light curve comprising forward shock emission only and the typical frequency of the reverse shock emission being shown to lie in the radio band as early as 137 seconds after the burst [60]. Alternatively, some optical flashes may be suppressed by strong magnetization.

Direct Observational Tests_Polarisation. Synchrotron radiation is intrinsically highly polarised; specific properties of the emitting region may reduce the observed degree of polarisation, (e.g., [63-71]), so detection of significant polarisation at early-time provides a direct signature of largescale ordered magnetic fields in the expanding fireball [72]. Claims of high levels of polarisation observed in the gammaray prompt emission have remained controversial: Coburn and Boggs [73] reported a high degree $P=80 \pm 20 \%$ of linear polarisation of the prompt emission of GRB 021206, but reanalysis of the data showed null polarisation $[74,75]$. In other cases, BATSE data of two bursts were reported to 

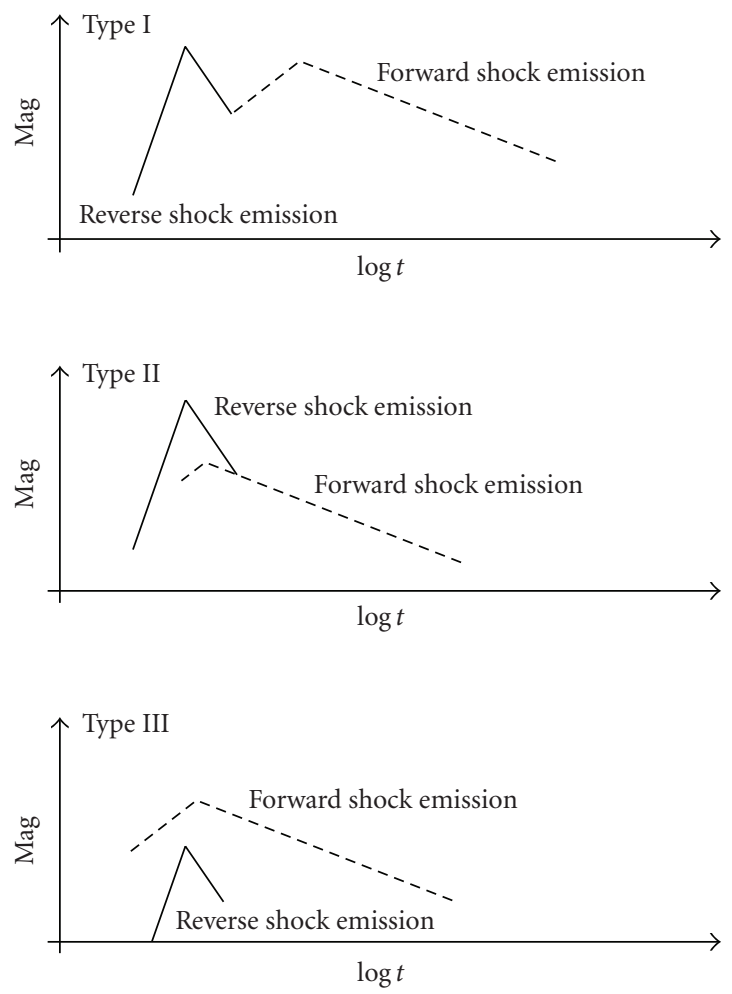

(a)

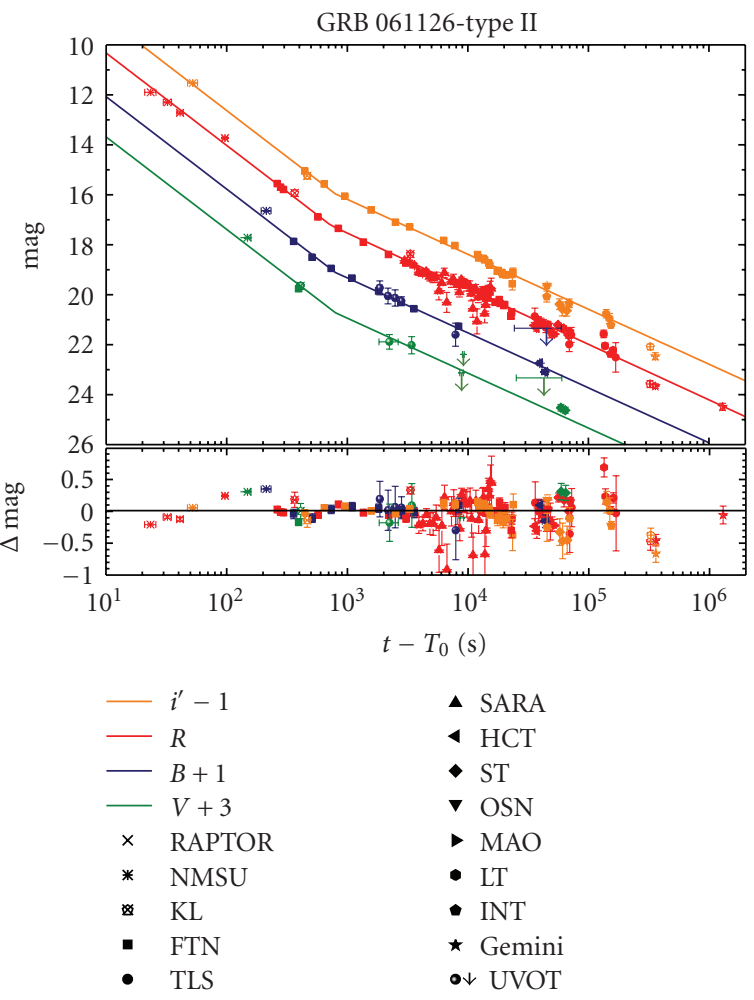

(b)

FIGURE 6: (a) Light-curves with different reverse-forward shock contributions. Magnetic energy density determines the relative contributions [61]. (b) Optical light curves of GRB 061126 showing the steep-to-shallow temporal break characteristic of a Type II reverse-forward shock observed after the reverse-shock peak [62].

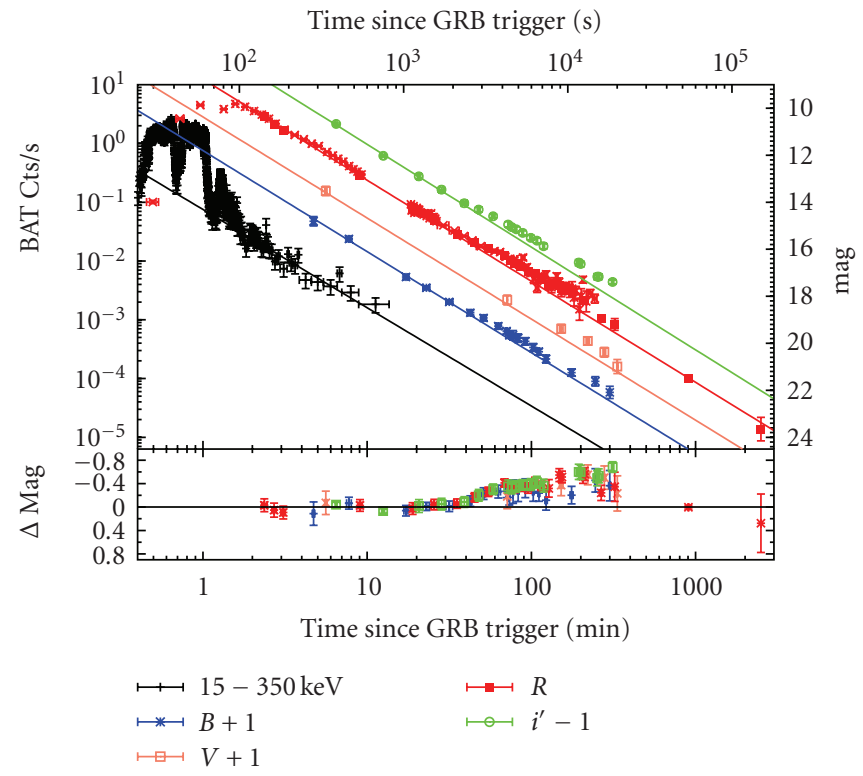

(a)

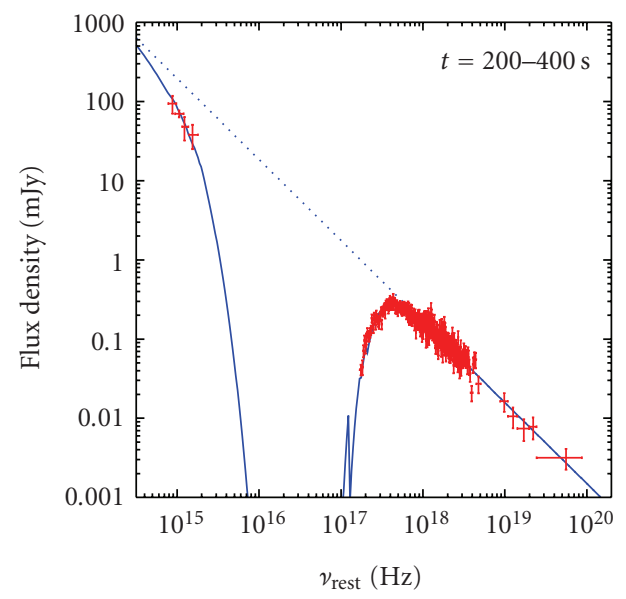

(b)

FIGURE 7: (a) $\gamma$-ray (black) and optical $B V R i^{\prime}$ (colour) light curves of GRB 061007 all decaying with a steep power-law index $\alpha \sim 1.7$ from 137 seconds to $\sim 10^{6}$ seconds. Despite its optical brightness $(R \sim 10.3$ mag at 137 seconds), a reverse-shock optical flash is ruled out (adapted from $[23,60])$. (b) Early-time $(200-400$ seconds) broad-band optical to $\gamma$-ray SED fitted with single absorbed power law $\beta$ (opt-X- $\gamma)=1.02$ \pm 0.05 and rest-frame extinction $A_{V}(\mathrm{SMC})=0.48 \pm 0.19 \mathrm{mag}$ (from [60]). 


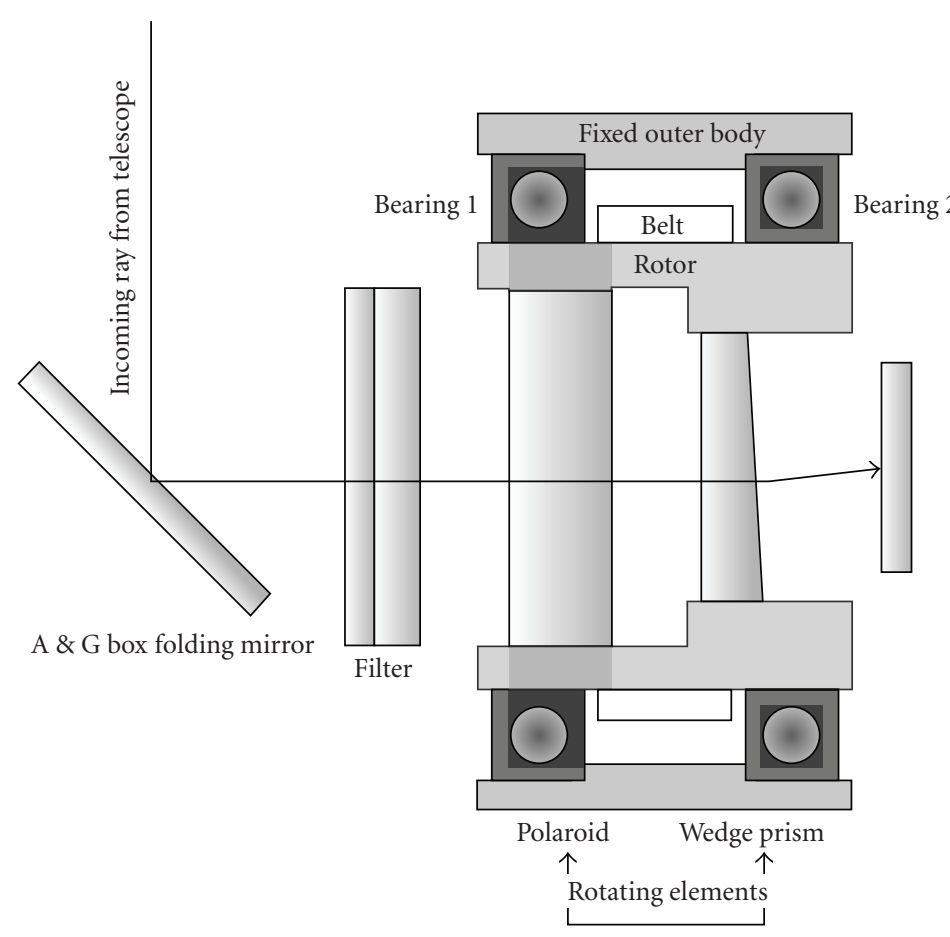

(a)
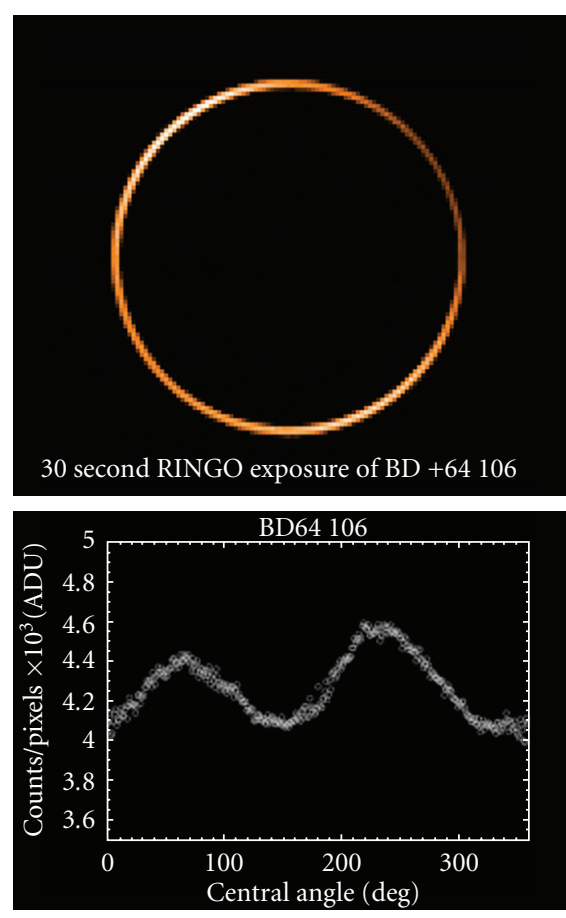

(b)

FIgure 8: (a) Diagram illustrating design of RINGO. (b) Polarised star BD +64 106 observed through RINGO (top panel): calibrated trace around ring showing polarisation signature (from [82]).

show evidence for $P>35 \%$ and $>50 \%$ using a GEANT4 model of the Earth albedo [76]. More recently, observations of GRB 041219A with the INTEGRAL imager IBIS show polarization up to $43 \%$ with rapid variability of degree and position angle [71], although independent confirmation with spectrometer SPI data remains difficult due to instrumental systematics [77, 78]. In the remaining cases, only upper limits to possible large polarisation degrees have been reported (e.g., [79]). Although careful calibration of instrumental systematics for $\gamma$-ray data remains challenging, these measurements show tantilising support for largescale ordered magnetic fields in the region responsible for the prompt $\gamma$-ray production. Measuring the early optical polarisation provides a direct probe of the magnetic field configuration. Optical polarisation observations of GRBs have the advantage that stars in the GRB field of view can be used as simultaneous independent checks on any instrumental systematics. Theoretical models of GRBs predict that mildly magnetized outflows produce strong reverse shock emission that should be polarised [51, 52, 68, 69, 80, 81], making early-time optical polarisation measurements vital for direct determination of the magnetic field structure.

The time-variable nature of optical emission from GRBs, however, makes traditional polarisation instruments unsuitable; we therefore designed the novel RINGO polarimeter on the LT [82], whose overall layout is shown in Figure 8. Its design was based on a novel ring polarimeter concept explored by Clarke and Neumayer [83]. This makes use of a rotating Polaroid to modulate the incoming polarised flux to be studied and is followed by deviating optics which corotate. A filter equivalent to $V+R$ bands was chosen to optimise the signal to noise ratio, as estimated from the typical GRB colours and to avoid the fringing on the CCD that the I filter would have caused. The result of this design is that each source image is recorded on the CCD as a ring, with the polarisation signal mapped out along the ring in a $\sin (2 \theta)$ pattern (Figure $8(\mathrm{~b}))$. One benefit of this design is the reduction of the saturation constraints on high precision photometry, as the flux is spread over the pixels along the ring. The potential problem of many rings overlapping with each other is only a concern for crowded fields which correlate with low galactic latitudes, that is, where GRB optical counterparts are strongly extinguished and therefore observationally already disfavoured. The design was optimised through requirements on (i) the rotation speed, to minimise impact on the polarisation ring profiles; and (ii) the geometry of the deflecting optics, to optimise the ring size. The polarimetric accuracy of RINGO is a few percents for an $R=15$-mag object in a 10 -second long exposure and decreases to a few $0.1 \%$ in a 10 -minute exposure. Further details on the RINGO polarimeter and its calibration and data reduction can be found in Steele et al. [82].

RINGO has now measured the optical polarisation of two GRBs (Figures 9, 10, and 11): Mundell et al. reported $P<8 \%$ in GRB 060418 at 203 seconds post burst [84] and Steele et al. 2009 measured $P=10.2 \pm 1.3 \%$ in GRB 090102 at 160.8 seconds post burst [85]. In the standard 


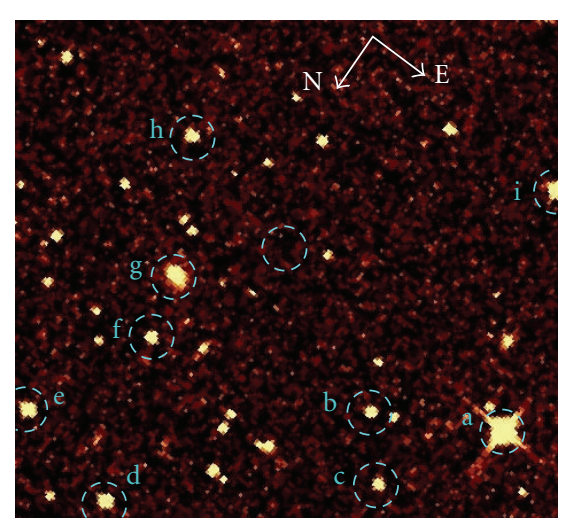

(a)

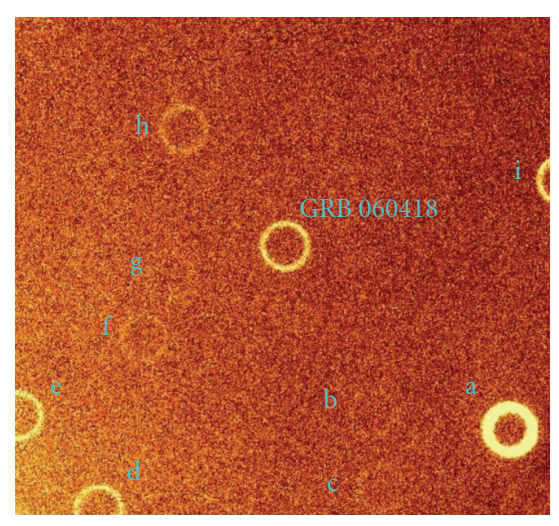

(b)

FIGURE 9: (a) DSS image of the field before the occurrence of GRB 060418. (b) RINGO image showing GRB 060418 and other field sources as rings (from [84]).

GRB fireball model both a forward shock, propagating into the circumburst medium, and a reverse shock, propagating into the GRB ejecta, contribute to the observed afterglow emission [61, 86] (Figure 6). The measurement in GRB 060418 was made close to the peak in the optical light curve at the time of deceleration of the fireball associated with the onset of the afterglow, when the reverse and forward shocks contributed equally to the detected light, while GRB 090102 was measured when the light curve was dominated by reverse-shock emission. Below we summarise the observations and suggest a unifying model to reconcile the two results.

Optical Polarisation Measurements of GRB 060418 and GRB 090102. The RINGO images of the fields containing GRB 060418 and 090102 are shown in Figures 9 and 10. GRB 060418 was detected by the Swift Burst Alert Telescope (BAT) on April 18, 2006 and showed a multipulsed gamma-ray profile with a total duration of 52 seconds, followed by a separate small bump at 130 seconds concomitant with a large X-ray flare observed with the Swift X-Ray Telescope (XRT) $[87,88]$ and probably due to prolonged internal activity (see Figure 11). A number of robotic facilities reacted to the prompt Swift alert and detected the optical and NIR counterpart: in particular, REM detected a smooth optical rise peaking at 153 seconds, interpreted as the onset of the afterglow [89], whereas the X-ray curve was seen to decline from the beginning, apart from the large flare mentioned above (Figure 11). The LT was triggered automatically, starting observations with a 30 -second long exposure taken with the RINGO polarimeter at 203 seconds post GRB, that is, simultaneously with the fading tail of the prompt gamma-ray emission (Figure 11) and the close to the X-ray flare. However, the contribution in the optical band from the X-ray flare was estimated to be negligible, thus confirming that RINGO measured the afterglow [84].

Swift detected GRB 090102 on 2 January 2009 at 02:55:45 UT, with a pulse of gamma rays lasting $T_{90}=27$ seconds and comprising four overlapping peaks starting 14-second before the trigger time [91]. The automatic localization was communicated to ground-based facilities, and a single 60second RINGO exposure was obtained starting 160.8 seconds after the burst. Simultaneous with our polarization observation of GRB 090102, a number of automated photometric followups were performed by other facilities [85]. The optical light curve (Figure 11), beginning at 40-second post burst, is fitted by a steep-shallow broken power law $\alpha_{1}=1.50 \pm 0.06$ and then $\alpha_{2}=0.97 \pm 0.03$ after approximately 1000 seconds [90]. In contrast, the X-ray light curve began at 396 seconds post burst and shows a steady decay consistent with a single power law with slope $\alpha=1.36 \pm 0.01$ and no evidence of flares or breaks up to $t>7 \times 10^{5}$ seconds [90]. The absence of additional emission components, for example, from latetime central engine activity, in the optical light curves of GRB 060418 and 090102 allows a straightforward interpretation of their early-time polarisation in the context of current GRB models.

Despite the brightness of the optical afterglow of GRB 060418, no dominant reverse shock was observed, similar to other cases in which the typical frequency is inferred to lie below the optical band at early-time such as GRB 061007 [60]. Instead, the light curve peak is typical of the forward shock. The forward shock peaks either when fireball decelerates or when the typical synchrotron frequency crosses the observed band: the latter was ruled out for GRB 060418, as no colour evolution was observed around the peak, thus supporting the deceleration interpretation with the synchrotron frequency lying below the NIR bands at the peak time [89]. This is also consistent with the steep $(|\alpha| \sim 2.7)$ rise observed in the NIR bands (Figure 11), in agreement with the theoretical expectations for the forward shock deceleration [92]. The RINGO measurement of GRB 060418 was made close to the deceleration time at the onset of the afterglow and when any magnetic field would still be present in the emitting region because the detected light contains an equal contribution from forward and reverse shocks, that is, Type III $[12,61]$ (Figure 6).

In contrast to GRB 060418, GRB 090102 exhibited the steep-shallow optical decay typical of that expected from an 


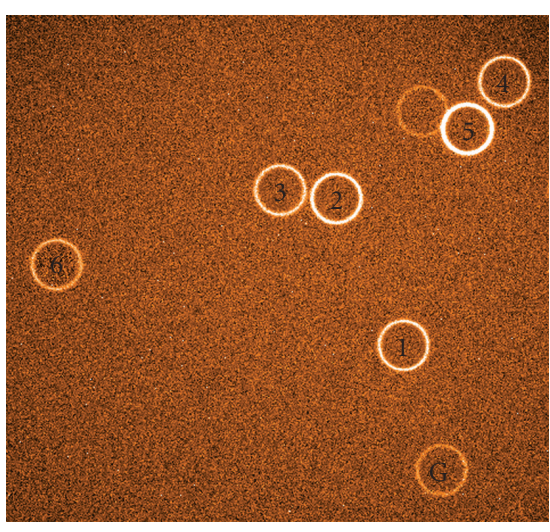

(a)
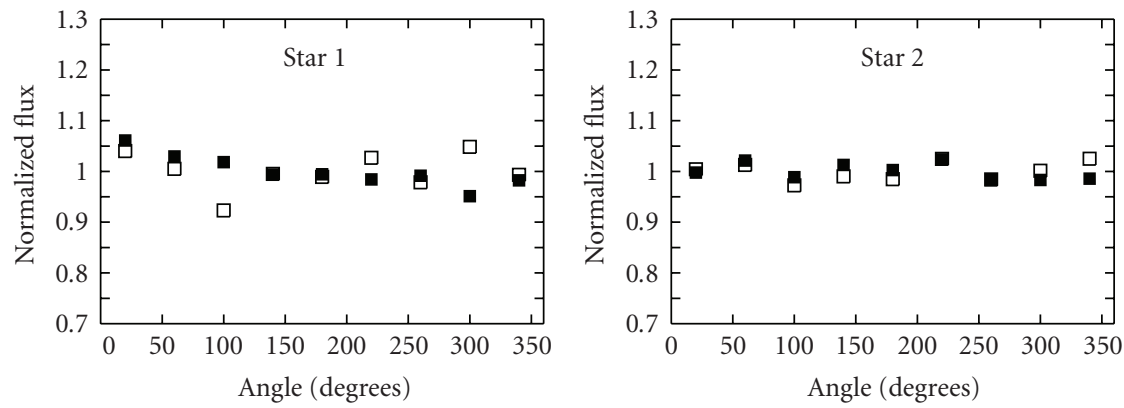

$09 / 05 / 19 P=2.5+/-0.2(\mathrm{sd})$

- $09 / 01 / 02 P=2.4+/-0.2(\mathrm{sd})$

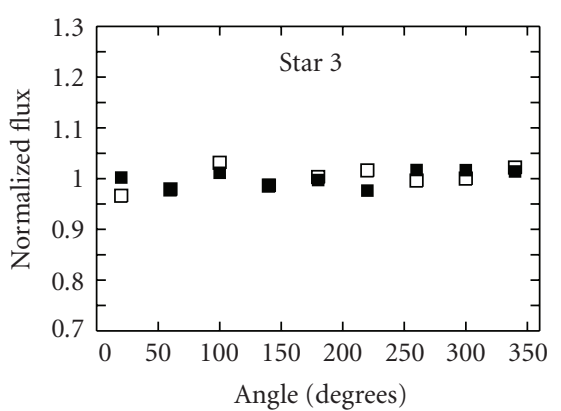

ㅁ $09 / 05 / 19 P=1.3+/-0.3(\mathrm{sd})$

- $09 / 01 / 02 P=1.5+/-0.2(\mathrm{sd})$

(b)

FIGURE 10: (a) RINGO image of field containing GRB 090102 (marked “G”); field stars are also visible. (b) Traces around rings for stars 1, 2, 3 and GRB 090102; derived polarisation \% for each is quoted. [85].

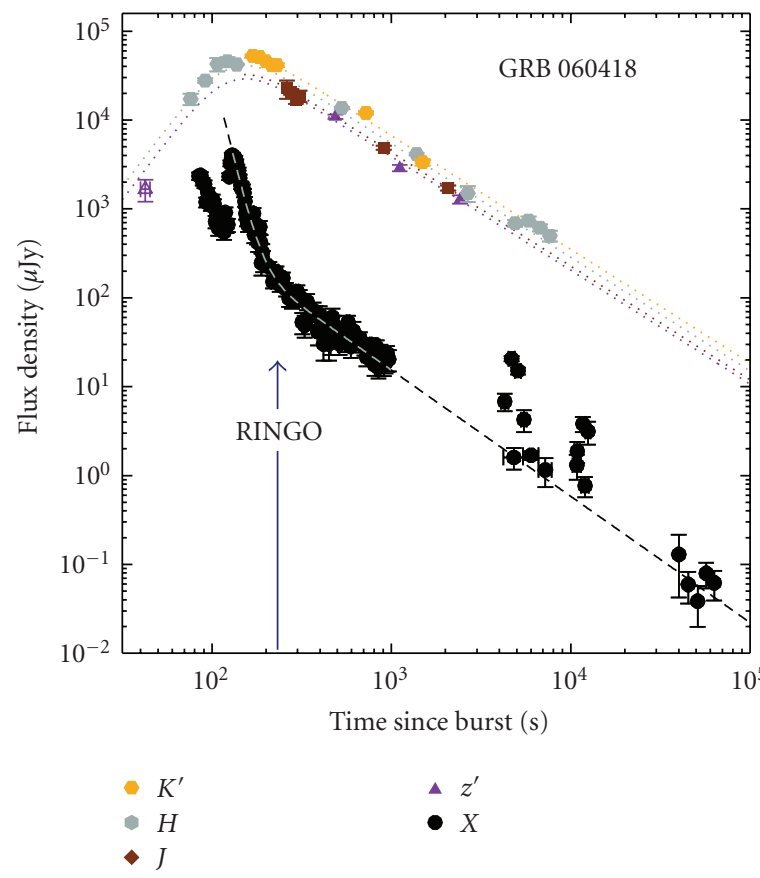

(a)

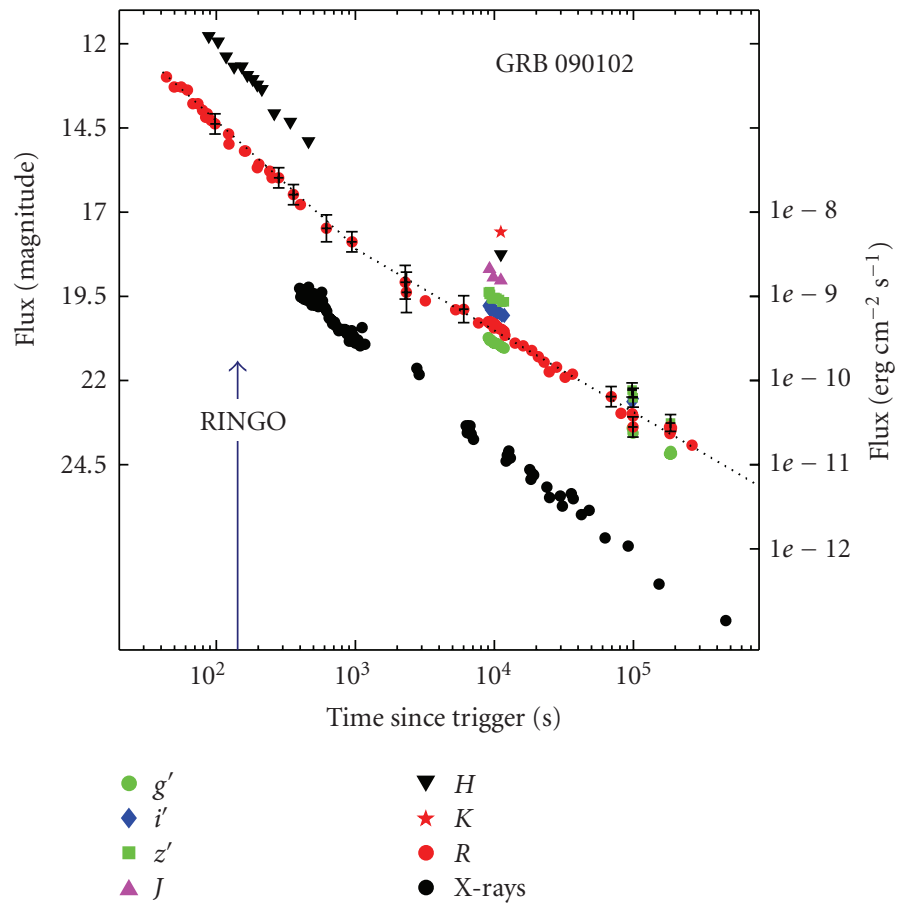

(b)

FIGURE 11: Light curves of GRB 060418 and GRB 090102 with time of RINGO observation marked at 203 seconds and 160 seconds (adapted from [89] and [90]), respectively. 


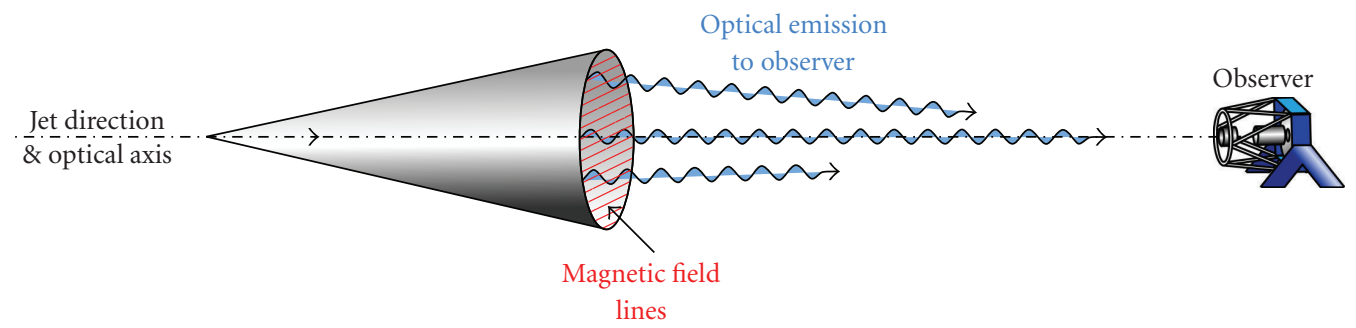

(a)

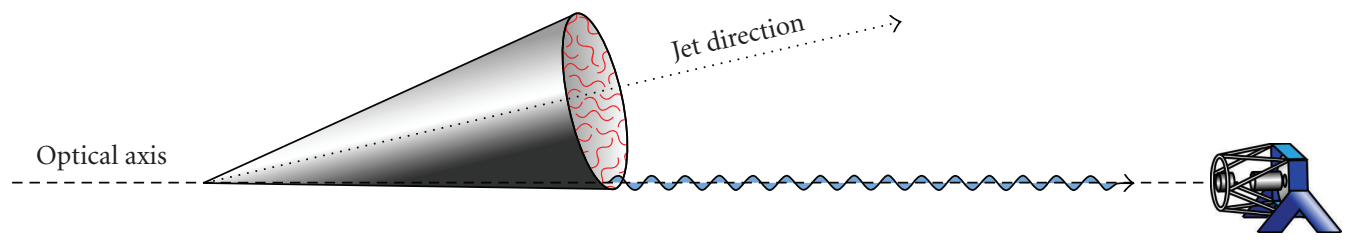

(b)

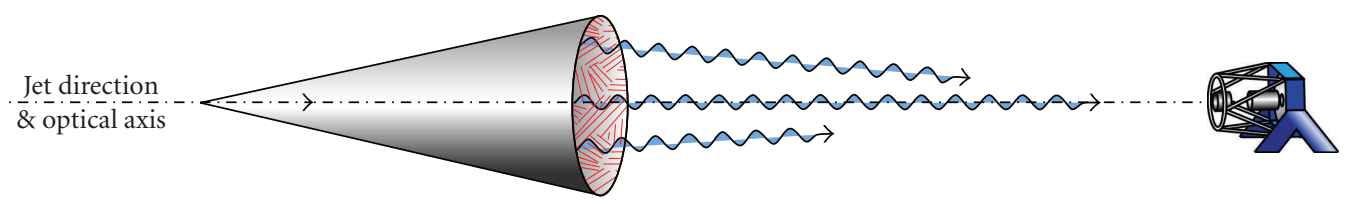

(c)

FIGURE 12: Competing models of GRB magnetic field structure for standard fireball model: (a) large-scale ordered magnetic field (favoured for GRB 090102); (b) tangled magnetic field in shock front and line of sight close to jet edge, and (c) shock front contains some independent patches of locally ordered magnetic fields [85].

afterglow whose emission is dominated by the reverse shock emission at early-times (Figure 11); this is the first GRB for which polarized optical light at early-time has been detected and its high level of polarisation $P=10.2 \pm 1.3 \%$ requires the presence of large-scale ordered magnetic fields in the outflow [85] (Figure 12). As the RINGO measurement was made when the reverse shock emission dominated the light curve, the large polarisation signal provides the first direct evidence that reverse shocks are produced in the presence of such fields.

Figure 12 shows three competing models of GRB magnetic field structure for the standard fireball model that could produce polarised light: (a) large-scale ordered magnetic field threaded through the GRB outflow-this model is favoured for GRB 090102; (b) tangled magnetic field generated in the shock front and an off-axis line-of-sight close to the jet edge - a scenario ruled out by the optical light curves for which an off-axis jet would require a shallowto-steep decay, where the opposite is observed in GRB 090102; (c) a shock front containing independent patches of locally ordered magnetic fields - a scenario that is unlikely to account for GRB 090102 because the maximum predicted polarisation is $10 \%$ under the extreme assumption that the coherence length grows at the speed of light in the local fluid frame after the field is generated.

In summary, the polarisation properties of GRB 060418 and GRB 090102 can be reconciled in a single model in which the outflow is mildly magnetised $(\sigma \sim 1)$ and contains large-scale ordered magnetic fields. In GRB 060418, $\sigma$ slightly larger than unity is needed to suppress the reverse shock emission (and hence polarisation), while in GRB 090102, $\sigma$ slightly less than unity will produce the bright polarised reverse shock emission that is observed.

\section{Summary and Prospects}

Exploring the extreme physics exhibited by GRB explosions is technically challenging due to (a) the unpredictability of their occurrence, (b) their short-lifetime rapidly fading emission, and (c) the wide range of observed brightnesses of optical counterparts, ranging from $R=5$ to $>22$ mag within minutes of the burst itself-all drivers of autonomous followup technology. Deep, fast, and multifilter observations are crucial to identify the counterparts to these events that represent the brightest stellar objects observed out to the epoch of reionization. With the advent of Swift, discoveries such as the canonical early X-ray decay, the X-ray flares, the detection of the afterglows of short-duration GRBs, and the recognition that GRB-producing stars exist out at least to $z=$ 8.2 keep GRB studies at the forefront of astrophysics.

Efforts continue to understand the complexity of the Xray versus optical afterglow temporal evolution, the circumburst environment properties, in particular the dust versus gas content around the GRB progenitor and along the line of sight through the host galaxy, and the origin(s) of optical flares and their possible interpretations (e.g., GRB 080129 [43]: residual collisions in the GRB outflow or hot spots in strongly magnetized ejecta? [93]). Questions remain on 
the fundamental nature of the relativistic ejecta, the underlying radiation mechanisms, and the role of magnetic fields. Observational surprises such as the relative lack of $\mathrm{GeV}$ emission from many bursts detected by Fermi and the rich variety in optical properties of GRB counterparts continue to drive developments in GRB modelling and observational technology. The $2 \mathrm{~m}$ robotic telescopes described here are proving decisive in tackling many of these issues: the variety of light curves has been investigated both on statistical grounds [12] as well as in individual cases of special interests: the dark GRB population and the luminosity function distribution have been characterised over a broad range of apparent brightnesses and the presence and lack of reverse shock emission in specific cases was investigated.

The interplay between forward and reverse shocks within the standard fireball model, as determined by the magnetic properties of the outflow, may yet succeed in explaining the dearth of reverse shocks previously expected from preSwift observations. The use of the RINGO polarimeter on the LT, capable of measuring the polarisation of optical counterparts to GRBs as early as a few minutes after the onset of the prompt $\gamma$-ray emission, has provided the earliest measurements and detection of GRB polarisation, setting important direct constraints on the magnetic field structure of the fireball and on the jet configuration [84, 85]. Further progress made in understanding the magnetic field structure of the fireball-large-scale ordered fields are currently preferred over locally tangled fields in the shock layer-will contribute to our knowledge of the nature of the outflow along the jets. Time-resolved early-time polarisation light curves (\% and PA) from the newly commissioned RINGO2 promise to provide unprecedented diagnostic information on the structure and evolution of the outflow and its magnetic field for a statistically significant sample of GRBs down to $R<17 \mathrm{mag}$ and thus allow powerful discrimination between predictions of the hydrodynamical versus magnetised jet models and ultimately constrain the physics of GRB central engines.

\section{Acknowledgments}

The authors thank the Liverpool Telescope group for technical, scientific, and artwork support. C. G. Mundell acknowledges financial support from Research Councils U.K. The Liverpool Telescope is located at the Observatorio del Roque de Los Muchachos, La Palma, Canary Islands, Spain. It was designed and built by Telescope Technologies Ltd and is owned and operated by Liverpool John Moores University with financial support from the U.K. PPARC. The Faulkes telescopes, owned by Las Cumbres Observatory, are located in Hawaii (FTN) and Siding Spring, Australia (FTS), and are operated with support from the Dill Faulkes Educational Trust.

\section{Endnotes}

1. Now owned by Las Cumbres Observatory, are operated with support from the Dill Faulkes Educational Trust.

\section{References}

[1] R. W. Klebesadel, I. B. Strong, and R. A. Olson, "Observations of gamma-ray bursts of cosmic origin," Astrophysical Journal, vol. 525 , no. 1, pp. 1214-1217, 1999.

[2] E. Costa, F. Frontera, J. Heise, et al., "Discovery of an X-ray afterglow associated with the $\gamma$-ray burst of 28 February 1997," Nature, vol. 387, no. 6635, pp. 783-785, 1997.

[3] J. Van Paradijs, P. J. Groot, T. Galama, et al., "Transient optical emission from the error box of the $\gamma$-ray burst of 28 february 1997," Nature, vol. 386, no. 6626, pp. 686-689, 1997.

[4] D. A. Frail, S. R. Kulkarnit, L. Nicastro, M. Feroci, and G. B. Taylor, "The radio afterglow from the $\gamma$-ray burst of 8 May 1997," Nature, vol. 389, no. 6648, pp. 261-263, 1997.

[5] N. Gehrels, G. Chincarini, P. Giommi, et al., "The Swift gamma-ray burst mission," Astrophysical Journal, vol. 611, no. 2, pp. 1005-1020, 2004.

[6] W. S. Paciesas, C. A. Meegan, G. N. Pendleton, et al., "The fourth BATSE gamma-ray burst catalog (revised)," Astrophysical Journal, Supplement Series, vol. 122, no. 2, pp. 465-495, 1999.

[7] S. Barthelmy, "GCN and VOEvent: a status report," Astronomische Nachrichten, vol. 329, no. 3, pp. 340-342, 2008.

[8] C. Akerlof, R. Balsano, S. Barthelmy, et al., "Observation of contemporaneous optical radiation from a $\gamma$-ray burst," Nature, vol. 398, no. 6726, pp. 400-402, 1999.

[9] G. Boella, R. C. Butler, G. C. Perola, L. Piro, L. Scarsi, and J. A. M. Bleeker, "BeppoSAX, the wide band mission for Xray astronomy," Astronomy and Astrophysics Supplement Series, vol. 122, no. 2, pp. 299-307, 1997.

[10] D. A. Frail, "Beaming in gamma-ray bursts: evidence for a standard energy reservoir," Astrophysical Journal, vol. 562, no. 1, pp. L55-L58, 2001.

[11] G. Ghirlanda, G. Ghisellini, D. Lazzati, and C. Firmani, "Gamma-ray bursts: NEW rulers to measure the universe," Astrophysical Journal, vol. 613, no. 1, pp. L13-L16, 2004.

[12] A. Melandri, C. G. Mundell, S. Kobayashi, et al., "The early-time optical properties of gamma-ray burst afterglows," Astrophysical Journal, vol. 686, no. 2, pp. 1209-1230, 2008.

[13] S. B. Cenko, J. Kelemen, F. A. Harrison, et al., "Dark bursts in the Swift era: the palomar 60 inch-Swift early optical afterglow catalog," The Astrophysical Journal, vol. 693, no. 2, pp. 1484-1493, 2009.

[14] S. R. Oates, M. J. Page, P. Schady, et al., "A statistical study of gamma-ray burst afterglows measured by the Swift Ultraviolet Optical Telescope," Monthly Notices of the Royal Astronomical Society, vol. 395, no. 1, pp. 490-503, 2009.

[15] S. A. Yost, F. Aharonian, C. W. Akerlof, et al., "The dark side of rotse-III prompt GRB observations," Astrophysical Journal, vol. 669, no. 2, pp. 1107-1114, 2007.

[16] P. W. A. Roming, P. Schady, D. B. Fox, et al., "Very early optical afterglows of gamma-ray bursts: evidence for relative paucity of detection," Astrophysical Journal, vol. 652, no. 2, pp. 14161422, 2006.

[17] G. Tagliaferri, M. Goad, G. Chincarini, et al., "An unexpectedly rapid decline in the X-ray afterglow emission of long $\gamma$-ray bursts," Nature, vol. 436, no. 7053, pp. 985-988, 2005.

[18] D. N. Burrows, P. Romano, A. Falcone, et al., "Astrophysics: bright X-ray flares in gamma-ray burst afterglows," Science, vol. 309, no. 5742, pp. 1833-1835, 2005.

[19] P. T. O’Brien, R. Willingale, J. Osborne, et al., "The early Xray emission from GRBs," Astrophysical Journal, vol. 647, no. 2, pp. 1213-1237, 2006. 
[20] G. Chincarini, A. Moretti, P. Romano, et al., "The first survey of X-ray flares from gamma-ray bursts observed by Swift: temporal properties and morphology," Astrophysical Journal, vol. 671, no. 2, pp. 1903-1920, 2007.

[21] A. D. Falcone, D. Morris, J. Racusin, et al., "The first survey of X-ray flares from gamma-ray bursts observed by Swift: spectral properties and energetics," Astrophysical Journal, vol. 671, no. 2, pp. 1921-1938, 2007.

[22] A. Klotz, M. Boër, J. L. Atteia, and B. Gendre, "Early optical observations of gamma-ray bursts by the TAROT telescopes: period 2001-2008," The Astronomical Journal, vol. 137, no. 5, pp. 4100-4108, 2009.

[23] E. S. Rykoff, F. Aharonian, C. W. Akerlof, et al., "Looking into the fireball: Rotse-III and Swift observations of early gammaray burst afterglows," Astrophysical Journal, vol. 702, no. 1, pp. 489-505, 2009.

[24] J. F. Graham, A. S. Fruchter, A. J. Levan, et al., "GRB 070714B: discovery of the highest spectroscopically confirmed short burst redshift," The Astrophysical Journal, vol. 698, no. 2, pp. 1620-1629, 2009.

[25] N. Kawai, G. Kosugi, K. Aoki, et al., "An optical spectrum of the afterglow of a $\gamma$-ray burst at a redshift of $z=6.295$," Nature, vol. 440, no. 7081, pp. 184-186, 2006.

[26] J. Greiner, T. Krühler, J. P. U. Fynbo, et al., "GRB 080913 at redshift 6.7," The Astrophysical Journal, vol. 693, no. 2, pp. 1610-1620, 2008.

[27] R. Salvaterra, M. Della Valle, S. Campana, et al., "GRB 090423 at a redshift of $z \approx 8.1$," Nature, vol. 461, no. 7268, pp. 1258 1260, 2009.

[28] N. R. Tanvir, D. B. Fox, A. J. Levan, et al., "A $\gamma$-ray burst at a redshift of $z \approx 8.2$," Nature, vol. 461, no. 7268, pp. 1254-1257, 2009.

[29] F. M. Zerbi, G. Chincarini, G. Ghisellini, et al., "The REM telescope: detecting the near infra-red counterparts of gammaray bursts and the prompt behavior of their optical continuum," Astronomische Nachrichten, vol. 322, no. 5-6, pp. 275285, 2001.

[30] W. T. Vestrand, K. Borozdin, D. J. Casperson, et al., "RAPTOR: closed-Loop monitoring of the night sky and the earliest optical detection of GRB 021211," Astronomische Nachrichten, vol. 325, no. 6-8, pp. 549-552, 2004.

[31] D. Reichart, M. Nysewander, J. Moran, et al., "PROMPT: panchromatic robotic optical monitoring and polarimetry telescopes," Nuovo Cimento della Societa Italiana di Fisica C, vol. 28, no. 4-5, pp. 767-770, 2005.

[32] S. A. Yost, F. Aharonian, C. W. Akerlof, et al., "Status of the ROTSE-III telescope network," Astronomische Nachrichten, vol. 327, no. 8, pp. 803-805, 2006.

[33] A. Klotz, M. Boër, J. Eysseric, et al., "Robotic observations of the sky with TAROT: 2004-2007," Publications of the Astronomical Society of the Pacific, vol. 120, no. 874, pp. 12981306, 2008.

[34] S. B. Cenko, D. B. Fox, D.-S. Moon, et al., "The automated palomar 60 inch telescope," Publications of the Astronomical Society of the Pacific, vol. 118, no. 848, pp. 1396-1406, 2006.

[35] J. S. Bloom, D. L. Starr, C.H. Blake, et al., "Autonomous observing and control systems for PAIRITEL, a $1.3 \mathrm{~m}$ infrared imaging telescope," in Astronomical Data Analysis Software and Systems XV, vol. 351 of ASP Conference Series, pp. 751-754, 2006.

[36] C. Guidorzi, A. Monfardini, A. Gomboc, et al., "The automatic real-time gamma-ray burst pipeline of the $2 \mathrm{~m}$ Liverpool Telescope," Publications of the Astronomical Society of the Pacific, vol. 118, no. 840, pp. 288-296, 2006.
[37] A. Nousek, C. Kouveliotou, D. Grupe, et al., "Evidence for a canonical gamma-ray burst afterglow light curve in the Swift XRT data," Astrophysical Journal, vol. 642, no. 1, pp. 389-400, 2006.

[38] B. Zhang, Y. Z. Fan, J. Dyks, et al., "Physical processes shaping gamma-ray burst X-ray afterglow light curves: theoretical implications from the Swift X-ray Telescope observations," The Astrophysical Journal, vol. 642, no. 1, pp. 354-370, 2006.

[39] C. D. Dermer, "Rapid X-ray declines and plateaus in Swift GRB light curves explained by a highly radiative blast wave," Astrophysical Journal, vol. 664, no. 1, pp. 384-396, 2007.

[40] C. D. Dermer, "Nonthermal synchrotron radiation from gamma-ray burst external shocks and the X-ray flares observed with Swift," Astrophysical Journal, vol. 684, no. 1, pp. 430-448, 2008.

[41] A. Monfardini, S. Kobayashi, C. Guidorzi, et al., "High-quality early-time light curves of GRB 060206: implications for gamma-ray burst environments and energetics," Astrophysical Journal, vol. 648, no. 2, pp. 1125-1131, 2006.

[42] C. Guidorzi, S. D. Vergani, S. Sazonov, et al., "GRB 070311: a direct link between the prompt emission and the afterglow," Astronomy \& Astrophysics, vol. 474, no. 3, pp. 793-805, 2007.

[43] J. Greiner, T. Krühler, S. McBreen, et al., "A strong optical flare before the rising afterglow of GRB 080129," The Astrophysical Journal, vol. 693, no. 2, pp. 1912-1919, 2009.

[44] T. Krühler, J. Greiner, S. McBreen, et al., "Correlated optical and X-ray flares in the afterglow of XRF 071031," Astrophysical Journal, vol. 697, no. 1, pp. 758-768, 2009.

[45] A. Melandri, C. Guidorzi, S. Kobayashi, et al., "Evidence for energy injection and a finetuned central engine at optical wavelengths in GRB 070419A," Monthly Notices of the Royal Astronomical Society, vol. 395, no. 4, pp. 1941-1494, 2009.

[46] C. Guidorzi, D. Bersier, A. Melandri, et al., "GRB 060927: Faulkes Telescope South observation," GRB Coordinates Network, Circular Service, 5633, 2006, http://gcn.gsfc.nasa.gov/ gcn3/5633.gcn3.

[47] A. E. Ruiz-Velasco, H. Swan, E. Troja, et al., "Detection of GRB 060927 at $z=5.47$ : implications for the use of gamma-ray bursts as probes of the end of the dark ages," Astrophysical Journal, vol. 669, no. 1, pp. 1-9, 2007.

[48] S. R. Oates, C. G. Mundell, S. Piranomonte, et al., "Anatomy of a dark burst: the afterglow of GRB 060108," Monthly Notices of the Royal Astronomical Society, vol. 372, no. 1, pp. 327-337, 2006.

[49] D. A. Kann, S. Klose, and A. Zeh, "Signatures of extragalactic dust in PRE-Swift GRB afterglows," Astrophysical Journal, vol. 641, no. 2, pp. 993-1009, 2006.

[50] A. Kann, S. Klose, B. Zhang, et al., "The afterglows of Swift-era gamma-ray bursts. I. Comparing pre-Swift and Swift long/soft (Type II) GRB optical afterglows," submitted, http://arxiv.org/abs/0712.2186.

[51] M. Lyutikov, V. I. Pariev, and R. D. Blandford, "Polarization of prompt gamma-ray burst emission: evidence for electromagnetically dominated outflow," Astrophysical Journal, vol. 597, no. 2, pp. 998-1009, 2003.

[52] Y. Z. Fan, D. M. Wei, and C. F. Wang, "The very early afterglow powered by ultra-relativistic mildly magnetized outflows," Astronomy \& Astrophysics, vol. 424, no. 2, pp. 477-484, 2004.

[53] Y. -Z. Fan, D. Xu, and D. -M. Wei, "Polarization evolution accompanying the very early sharp decline of gamma-ray burst X-ray afterglows," Monthly Notices of the Royal Astronomical Society, vol. 387, no. 1, pp. 92-96, 2008.

[54] B. Zhang and S. Kobayashi, "Gamma-ray burst early afterglows: reverse shock emission from an arbitrarily magnetized 
ejecta," Astrophysical Journal, vol. 628, no. 1, pp. 315-334, 2005.

[55] T. Piran, "Gamma-ray bursts and the fireball model," Physics Report, vol. 314, no. 6, pp. 575-667, 1999.

[56] B. Zhang and P. Mészáros, "Gamma-ray bursts: progress, problems \& prospects," International Journal of Modern Physics A, vol. 19, no. 15, pp. 2385-2472, 2004.

[57] P. Mészáros, "Gamma-ray bursts," Reports on Progress in Physics, vol. 69, no. 8, pp. 2259-2321, 2006.

[58] B. Zhang, "Gamma-ray bursts in the Swift era," Chinese Journal of Astronomy and Astrophysics, vol. 7, no. 1, pp. 1-50, 2007.

[59] T. Piran and Y. -Z. Fan, "Gamma-ray burst theory after Swift," Philosophical Transactions of the Royal Society A, vol. 365, no. 1854, pp. 1151-1162, 2007.

[60] C. G. Mundell, A. Melandri, C. Guidorzi, et al., "The remarkable afterglow of GRB 061007: implications for optical flashes and GRB fireballs," Astrophysical Journal, vol. 660, no. 1, pp. 489-495, 2007.

[61] A. Gomboc, S. Kobayashi, C. G. Mundell, et al., "Optical flashes, reverse shocks and magnetization," in Proceedings of the 6th Huntsville Symposium on Gamma-Ray Bursts, vol. 1133 of AIP Conference Proceedings, pp. 145-150, 2009.

[62] A. Gomboc, S. Kobayashi, C. Guidorzi, et al., "Multiwavelength analysis of the intriguing GRB 061126: the reverse shock scenario and magnetization," Astrophysical Journal, vol. 687, no. 1, pp. 443-455, 2008.

[63] R. Sari, "Linear polarization and proper motion in the afterglow of beamed gamma-ray bursts," Astrophysical Journal, vol. 524, no. 1, pp. L43-L46, 1999.

[64] A. Gruzinov and E. Waxman, "Gamma-ray burst afterglow: polarization and analytic light curves," Astrophysical Journal, vol. 511, no. 2, pp. 852-861, 1999.

[65] G. Ghisellini and D. Lazzati, "Polarization light curves and position angle variation of beamed gamma-ray bursts," Monthly Notices of the Royal Astronomical Society, vol. 309, no. 1, pp. L7-L11, 1999.

[66] E. Waxman, "Astronomy: new direction for $\gamma$-rays," Nature, vol. 423, no. 6938, pp. 388-389, 2003.

[67] J. Greiner, S. Klose, K. Reinsch, et al., "Evolution of the polarization of the optical afterglow of the $\gamma$-ray burst GRB030329," Nature, vol. 426, no. 6963, pp. 157-159, 2003.

[68] J. Granot and A. Königl, "Linear polarization in gamma-ray bursts: the case for an ordered magnetic field," Astrophysical Journal, vol. 594, no. 2, pp. L83-L87, 2003.

[69] E. M. Rossi, D. Lazzati, J. D. Salmonson, and G. Ghisellini, "The polarization of afterglow emission reveals $\gamma$-ray bursts jet structure," Monthly Notices of the Royal Astronomical Society, vol. 354, no. 1, pp. 86-100, 2004.

[70] A. Sagiv, E. Waxman, and A. Loeb, "Probing the magnetic field structure in gamma-ray bursts through dispersive plasma effects on the afterglow polarization," Astrophysical Journal, vol. 615, no. 1, pp. 366-377, 2004.

[71] D. Götz, P. Laurent, F. Lebrun, F. Daigne, and E. Bonjak, "Variable polarization measured in the prompt emission of GRB 041219a using ibis on board integral," Astrophysical Journal, vol. 695, no. 2, pp. L208-L212, 2009.

[72] D. Lazzati, S. Covino, J. Gorosabel, et al., "On the jet structure and magnetic field configuration of GRB 020813," Astronomy \& Astrophysics, vol. 422, no. 1, pp. 121-128, 2004.

[73] W. Coburn and S. E. Boggs, "Polarization of the prompt $\gamma$-ray emission from the $\gamma$-ray burst of 6 December 2002," Nature, vol. 423, no. 6938, pp. 415-417, 2003.
[74] R. E. Rutledge and D. B. Fox, "Re-analysis of polarization in the $\gamma$-ray flux of GRB 021206," Monthly Notices of the Royal Astronomical Society, vol. 350, no. 4, pp. 1288-1300, 2004.

[75] C. Wigger, W. Hajdas, K. Arzner, M. Güdel, and A. Zehnder, "Gamma-ray burst polarization: limits from RHESSI measurements," Astrophysical Journal, vol. 613, no. 2, pp. 1088 1100, 2004.

[76] D. R. Willis, E. J. Barlow, A. J. Bird, et al., "Evidence of polarisation in the prompt gamma-ray emission from GRB 930131 and GRB 960924," Astronomy \& Astrophysics, vol. 439, no. 1, pp. 245-253, 2005.

[77] S. McGlynn, D. J. Clark, A. J. Dean, et al., "Polarisation studies of the prompt gamma-ray emission from GRB 041219a using the spectrometer aboard INTEGRAL," Astronomy \& Astrophysics, vol. 466, no. 3, pp. 895-904, 2007.

[78] E. Kalemci, S. E. Boggs, C. Kouveliotou, M. Finger, and M. G. Baring, "Search for polarization from the prompt gammaray emission of GRB 041219a with SPI on INTEGRAL," Astrophysical Journal, Supplement Series, vol. 169, no. 1, pp. 75-82, 2007.

[79] S. McGlynn, S. Foley, B. McBreen, et al., "High energy emission and polarisation limits for the INTEGRAL burst GRB 061122," Astronomy \& Astrophysics, vol. 499, no. 2, pp. 465-472, 2009.

[80] Y. Z. Fan, B. Zhang, and D. M. Wei, "Early optical-infrared emission from GRB 041219a: neutron-rich internal shocks and a mildly magnetized external reverse shock," Astrophysical Journal, vol. 628, no. 1, pp. L25-L28, 2005.

[81] S. Covino, "A closer look at a gamma-ray burst," Science, vol. 315, no. 5820, pp. 1798-1799, 2007.

[82] I. A. Steele, S. D. Bates, D. Carter, et al., "RINGO: a novel ring polarimeter for rapid GRB followup," in Ground-Based and Airborne Instrumentation for Astronomy, vol. 6269 of Proceedings of SPIE, Orlando, Fla, USA, May 2006.

[83] D. Clarke and D. Neumayer, "Experiments with a novel CCD stellar polarimeter," Astronomy \& Astrophysics, vol. 383, no. 1, pp. 360-366, 2002.

[84] C. G. Mundell, I. A. Steels, R. J. Smith, et al., "Early optical polarization of a gamma-ray burst afterglow," Science, vol. 315, no. 5820, pp. 1822-1824, 2007.

[85] I. A. Steele, C. G. Mundell, R. J. Smith, S. Kobayashi, and C. Guidorzi, "Ten per cent polarized optical emission from GRB 090102," Nature, vol. 462, no. 7274, pp. 767-769, 2009.

[86] S. Kobayashi, T. Piran, and R. Sari, "Hydrodynamics of a relativistic fireball: the complete evolution," Astrophysical Journal, vol. 513, no. 2, pp. 669-678, 1999.

[87] A. D. Falcone, S. D. Barthelmy, D. N. Burrows, et al., "GRB 060418: Swift detection of a burst with bright X-ray and optical afterglow," GRB Coordinates Network, Circular Service, 4966, 2006, http://gcn.gsfc.nasa.gov/gcn3/4966.gcn3.

[88] A. D. Falcone, D. N. Burrows, and J. Kennea, "GRB 060418: Swift XRT team refined analysis," GRB Coordinates Network, Circular Service, 4973, 2006, http://gcn.gsfc.nasa.gov/ gcn3/4973.gcn3.

[89] E. Molinari, S. D. Vergani, D. Malesani, et al., "REM observations of GRB060418 and GRB 060607A: the onset of the afterglow and the initial fireball Lorentz factor determination," Astronomy \& Astrophysics, vol. 469, no. 1, pp. L13-L16, 2007.

[90] B. Gendre, A. Klotz, E. Palazzi, et al., "Testing GRB models with the strange afterglow of GRB 090102," Monthly Notices of the Royal Astronomical Society,http://bit.ly/cvqXlG, in press. 
[91] T. Sakamoto, S. D. Barthelmy, W. H. Baumgartner, et al., "GRB 090102: Swift-BAT refined analysis,” GRB Coordinates Network, Circular Service, 8769, 2009, http://gcn.gsfc.nasa.gov/ gcn3/8769.gcn3.

[92] S. Kobayashi and B. Zhang, "GRB 021004: reverse shock emission," Astrophysical Journal, vol. 582, no. 2, pp. L75-L78, 2003.

[93] M. Lyutikov, "The electromagnetic model of gamma-ray bursts," New Journal of Physics, vol. 8, article 119, 2006. 

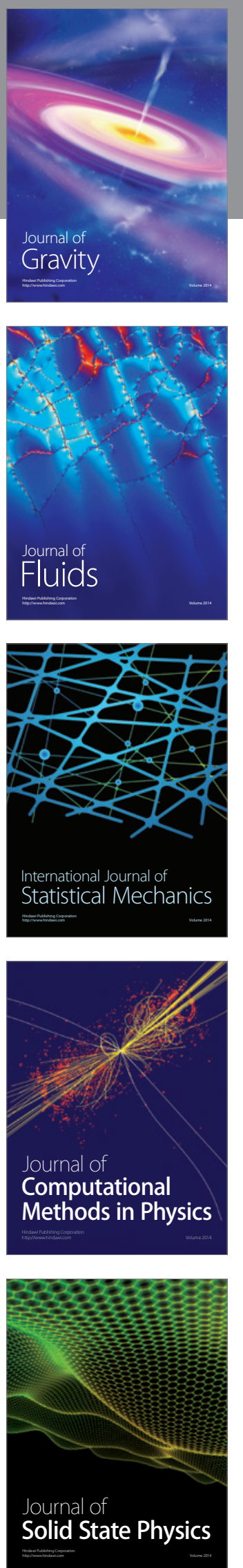

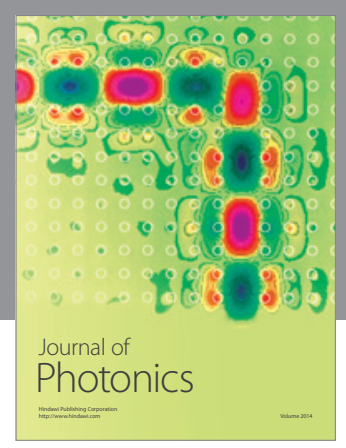

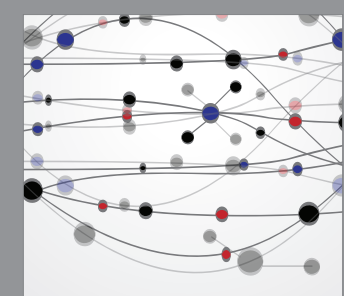

The Scientific World Journal
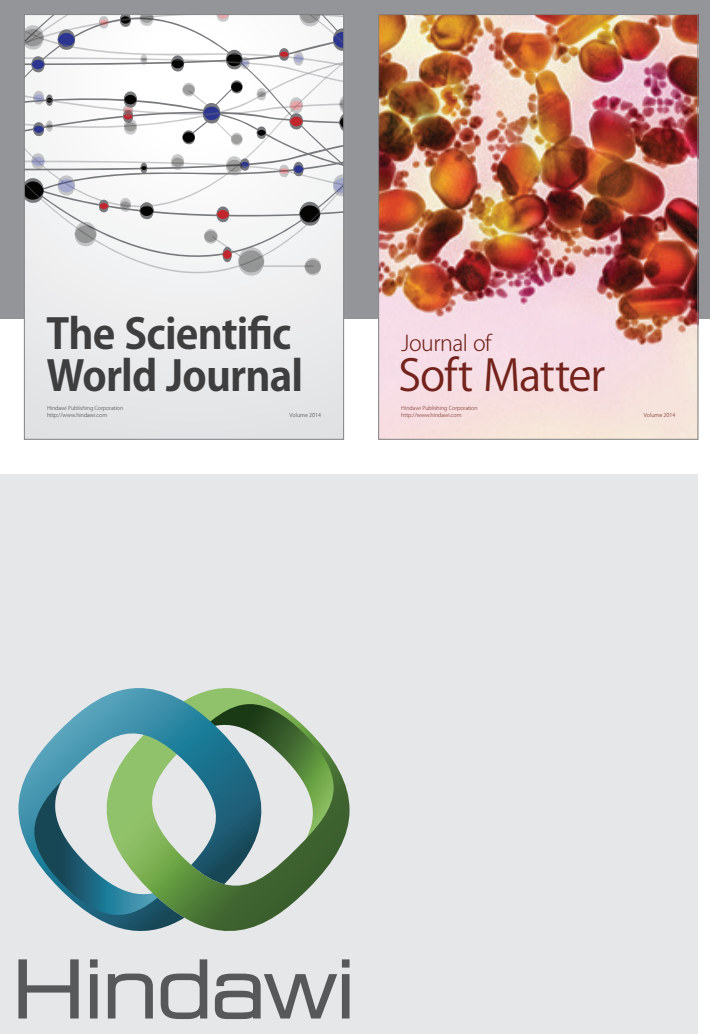

Submit your manuscripts at

http://www.hindawi.com
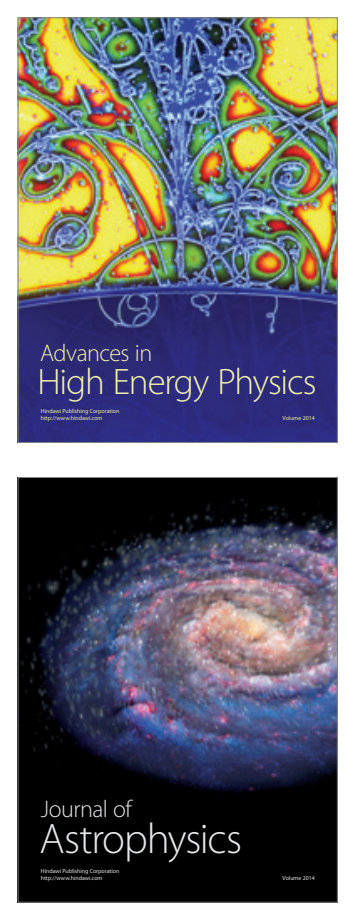
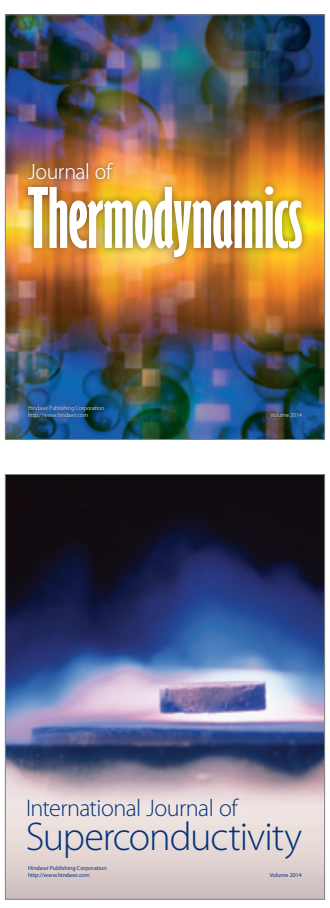
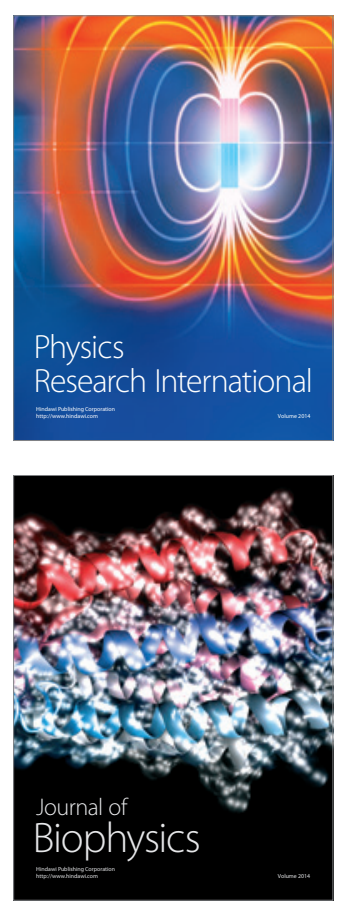
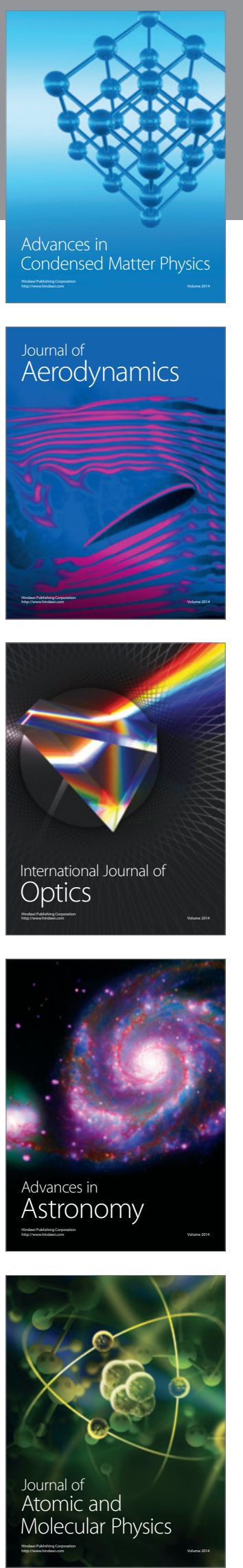